\title{
Pratiques de l'approvisionnement et de la commercialisation de la poule locale au Sénégal
}

\author{
Grégoire NAHIMANA ${ }^{1,3^{*}}$, Walter OSSEBI ${ }^{2}$, Ayao MISSOHOU ${ }^{3}$, Simplice Bosco \\ AYSSIWEDE $^{3}$, Paly CISSE ${ }^{4}$, Joseph BUTORE ${ }^{1}$ et Alioune TOURE ${ }^{4}$ \\ ${ }^{I}$ Faculté d'Agronomie et de Bio Ingénierie, Université du Burundi, BP 1550 Bujumbura, Burundi. \\ ${ }^{2}$ Service d'Economie et Gestion, Ecole Inter-Etats des Sciences et Médecine Vétérinaires de Dakar (EISMV), \\ BP 5077 Dakar, Sénégal. \\ ${ }^{3}$ Service de Zootechnie-Alimentation, Ecole Inter-Etats des Sciences et Médecine Vétérinaires de Dakar \\ (EISMV), BP 5077 Dakar, Sénégal. \\ ${ }^{4}$ Projet de Développement de l'Elevage au Sénégal oriental et en Haute Casamance (PDESOC), BP 176 \\ Tambacounda, Sénégal. \\ "Auteur correspondant ; E-mail : gregoirenahi@yahoo.fr
}

\section{RESUME}

Afin de caractériser le système d'approvisionnement et de commercialisation des poules locales, une enquête a été réalisée sur 122 acteurs de cette filière choisis au hasard dans la région de Kédougou au Sénégal. Les résultats ont montré que les enquêtés étaient à majorité des hommes ayant une expérience moyenne de 7,21 ans. Les sept acteurs identifiés suivaient un circuit court et/ou long dans un système informel. Les prix ont varié suivant l'acheteur, la période et la localité. Le plus utilisé des quatre modes de transport (véhicule, moto, vélo, à pied) identifiés a été celui à bicyclette. Seulement le panier et le cageot sont utilisés dans l'emballage. Les revenus ont varié d'un département et d'un acteur à l'autre, et ont permis d'assurer les dépenses familiales et de réaliser quelques économies. La commercialisation des poules locales a été plus rentable pour le grossiste rural comparé aux autres acteurs avec une valeur plus élevée dans le département de Saraya. Les principales difficultés identifiées ont été l'irrégularité du produit et l'enclavement de la zone. Il ressort de cette étude que l'amélioration des systèmes de production et de commercialisation pourrait constituer une stratégie de développement socio-économique et un outil de lutte contre la pauvreté en milieu rural.

(C) 2018 International Formulae Group. All rights reserved

Mots clés : Approvisionnement, commercialisation, poule locale, Sénégal.

\section{Supply and marketing of local chicken practices in Senegal}

\begin{abstract}
In order to characterize the supply and marketing system for local hens, a survey was conducted on 122 actors in the Kedougou region of Senegal. The results showed that the majority of traders were men with 7.21 years old experience. The seven kinds of chicken traders identified followed a short and / or long circuit in an informal system. The prices have varied according to the kind of actor, the period and locality. The bicycle
\end{abstract}


transport mode has largely been used of the four modes of transport (vehicle, motorcycle, bicycle, on foot). The basket and crate are only used in the package. Revenues have varied from one department and actor to another, and used to face the different family expenses and make some savings. The marketing of local hens was more profitable for the rural wholesaler compared to other actors with a higher value in the Saraya department. The major constraints identified were the irregularity of the product and the area remoteness. It appears from this study that improving production and marketing systems could be the socio-economic development strategy and a tool against rural poverty.

(C) 2018 International Formulae Group. All rights reserved.

Keywords: Supply, marketing, local chicken, Senegal.

\section{INTRODUCTION}

Au Sénégal, le sous-secteur de l'élevage avait contribué en 2012 à hauteur de $4,2 \%$ en moyenne à la formation du PIB du secteur primaire et national. Sa valeur ajoutée avait atteint plus de 300 milliards de Fcfa aux prix courants en 2012 (Niang et Mbaye, 2013). En cette même année, la population de volaille familiale était estimée à 23,929 millions de têtes, soit $40 \%$ de l'effectif total du cheptel national et sa production à 27847 tonnes, soit $14,49 \%$ de la production nationale de viande et d'abats (Niang et Mbaye, 2013). L'aviculture familiale est un levier pour améliorer les revenus et la sécurité alimentaire des ménages pauvres et démunis (Dieye et al., 2010 ; Emuron et al., 2010 ; Issa et al., 2012). Elle est la source de revenus la plus facile à mobiliser pour régler les besoins de l'unité familiale (Mwalusanya et al., 2002 ; Gondwe et al., 2005; Aklilu et al., 2007 ; Kugonza et al., 2008 ; Dieye et al., 2010 ; Emuron et al., 2010) et les recettes générées représentent une part très importante du budget des ménages ruraux (Traoré, 2005; Seye, 2007). Par ailleurs, chaque fois que les ménages ruraux ont un besoin d'argent, ils vendent les poulets indigènes (Okot, 1990) et les revenus issus de cette vente sont utilisés dans l'achat des biens de consommation pour le bien-être familial (Aklilu et al., 2007). Tenant compte de l'importance de ce sous-secteur avicole, la compréhension de tous les différents aspects de production et dynamiques commerciales et sociales qui y sont liés devient une nécessité absolue dans le but d'élaborer des stratégies et options pour son développement.

Malheureusement, même si l'aviculture familiale a fait l'objet de plusieurs études, très peu ont abordé le système de commercialisation. Les quelques études faites au Sénégal (Traoré, 2001 ; Dieye et al., 2010 ; Teno, 2010; Ossebi, 2011; Sikagueng Mbouga, 2011) ont montré que la commercialisation du poulet du pays est une filière mal organisée même si elle contribue à la sécurité alimentaire et à la réduction de la pauvreté. Par ailleurs, Dieye et al. (2010) ont rapporté que la vente de la volaille contribue en moyenne à $135 \%$ du revenu agricole total des exploitations et de $75,2 \%$ des revenus annuels d'élevage. Or, il est difficile de planifier et d'appliquer les programmes de développement de ce sous-secteur de l'élevage lorsqu'on ne maîtrise pas son système de production et de commercialisation (Hellin et al., 2005 ; Ossebi, 2011 ; Issa et al., 2012). C'est dans ce cadre que cette étude a été réalisée pour caractériser le système d'approvisionnement et de commercialisation du poulet du pays dans la région de Kédougou au Sénégal oriental. 


\section{MATERIEL ET METHODES}

\section{Période et milieu d'étude}

L'étude a été menée de juillet à septembre 2015 dans la Région de Kédougou située au Sénégal oriental (750 km de Dakar). Cette région est caractérisée par un climat subguinéen réparti en une saison de pluies unique et courte (fin mai à septembre), et une saison sèche longue (octobre à mai). La température moyenne est de $28{ }^{\circ} \mathrm{C}$ avec des précipitations annuelles variant de 600 à 1500 $\mathrm{mm}$ de pluies.

\section{Collecte des données}

L'enquête a porté sur un échantillon de 122 acteurs de la filière poulets du pays choisis au hasard dans toute la région auxquels un questionnaire d'enquête préétabli, testé et validé a été soumis. L'enquête s'est déroulée sur le lieu d'approvisionnement et/ou de commercialisation des poulets du pays. Les informations collectées au cours de cette enquête transversale comprenaient :

-les caractéristiques socioéconomiques du vendeur (sexe, âge, niveau de scolarisation, ethnie, raison et statut de l'activité, activité principale antérieure et cause de changement de l'activité, ancienneté et organisation du travail).

-les caractéristiques des pratiques d'achat et d'approvisionnement (lieu, critères et fréquence d'achat, différentes dépenses liées à l'achat et au transport et leur mode de règlement, technique d'emballage, moyen de déplacement, ...) ;

-les caractéristiques des pratiques de la vente (critères et fréquence de vente, différentes dépenses liées à la vente et leur mode de règlement, moyen de déplacement, ...);

-les contraintes relatives à l'achat et à la vente.

\section{Analyse des données}

Les informations recueillies ont été synthétisées en termes de pourcentages et moyennes avec des écarts types à l'aide du logiciel Statiscal Package for the Social Science (SPSS 16.0). L'analyse de la variance été faite pour comparer les moyennes. Les différents coûts liés aux dépenses (alimentation des poulets, transport, taxe municipale et de transport, frais de gardiennage, ...) ont été calculés. La marge bénéficiaire nette $(\mathrm{MBN})$ concernant les poulets a été calculée en prenant en compte les divers coûts à travers la relation : $\mathrm{MBN}=\mathrm{PV}-(\mathrm{PA}+$ diverses charges liées à l'activité) où $\mathrm{PV}$ et $\mathrm{PA}$ représentent, respectivement, les prix de vente et d'achat.

\section{RESULTATS \\ Caractéristiques socioéconomiques des vendeurs des poulets du pays}

Les vendeurs des poulets du pays sont majoritairement composés d'hommes $(90,2 \%)$ et situés dans la classe d'âge de 36 à 55 ans $(52,5 \%)$ avec une moyenne de $38,75 \pm 2,87$ ans. Ils sont pour la plupart musulmans $(97,5 \%)$ avec un niveau d'instruction assez bas ( $68,8 \%$ d'analphabètes et alphabétisés). Les activités antérieures au commerce des poulets du pays étaient généralement constituées de l'élevage (49,2\%) et de l'agro-élevage (29,5\%). Les principales raisons qui ont motivé le changement d'activités sont le fait que le commerce des poulets locaux est une source importante de revenus $(55,7 \%)$ et un moyen de subsistance (32\%) des personnes interrogées. La plus grande partie des enquêtés $(79,51 \%)$ a une expérience de 10 ans au plus avec un maximum de 40 ans et une moyenne de $7,21 \pm 6,95$ ans. La majeure partie des acteurs enquêtés sont plus représentés dans le département de Kédougou (50,82\%) et sont constitués des intermédiaires I $(35,25 \%)$ et II $(29,51 \%)$. Les grossistes urbains $(3,28 \%)$ ne sont rencontrés qu'à Kédougou seulement (Tableau 1). La plupart d'entre eux travaillent seuls et à leur propre compte $(89,3 \%)$. 


\section{Approvisionnement et commercialisation Typologie des acteurs}

Les acteurs, les niveaux, les prix, les différents circuits de commercialisation ainsi que le type de marché sont représentés sur la Figure 1. Les acteurs sont constitués des producteurs, des intermédiaires I et II, des grossistes ruraux et urbains, des détaillants et des consommateurs. L'intermédiaire I, qualifié aussi de villageois, collecte les poulets auprès des producteurs qu'il cède à l'intermédiaire II ou aux grossistes ruraux. L'intermédiaire II s'approvisionne auprès des producteurs et des collecteurs villageois. Il vend les poulets aux grossistes ruraux ou urbains, aux détaillants ou aux consommateurs. Ces intermédiaires I et II, qui parcourent des villages et des marchés ruraux pour acheter des poulets auprès des producteurs, utilisent leurs propres capitaux (mode direct) ou agissent en tant qu'intermédiaires au service des grossistes (mode indirect). Le grossiste rural, qui s'approvisionne auprès des producteurs et des intermédiaires I et II aux marchés ruraux, commercialise ses poulets aux grossistes urbains, détaillants ou aux consommateurs du marché urbain. Le grossiste urbain vend aux détaillants et quelque fois aux consommateurs les poulets achetés aux intermédiaires II et aux grossistes ruraux. Les détaillants se positionnent toujours sur le marché rural ou urbain et s'approvisionnent soit auprès des grossistes ruraux ou urbains soit aux intermédiaires II. Ces détaillants livrent leurs produits uniquement aux consommateurs (ménages). Toutefois, les consommateurs peuvent acheter aussi des poulets apportés au marché par des producteurs.

\section{Critères recherchés par les acteurs ou de fixation des prix}

D'après les personnes interrogées, les critères de fixation de prix d'achat sont le poids/taille de l'oiseau $(35,79 \%)$, le sexe $(22,37 \%)$ et la couleur du plumage $(11,84 \%)$.
Pour la vente, à ces trois critères représentés, respectivement, par $30,61 \%, 19,83 \%$ et $18,95 \%$ s'ajoute le prix d'achat $(30,61 \%)$. Les mêmes critères sont également recherchés par les clients avec une prédominance du couple poids et sexe pour les ménages $(57,87 \%$ et $35,65 \%)$, les détaillants $(64,71 \%$ et $33,69 \%)$, les restaurateurs $(67,4 \%$ et $32,04 \%)$ et les grossistes $(65,59 \%$ et $33,33 \%)$. Par contre, les sacrificateurs fondent leur choix principalement sur la couleur du plumage $(58,76 \%)$ et le sexe $(31,96 \%)$.

Les résultats révèlent que $93,4 \%$ des acteurs n'ont pas conclu de contrat avec leurs fournisseurs. Les causes importantes évoquées sont la méconnaissance de contrat $(40,35 \%)$, l'irrégularité des fournisseurs $(24,56 \%)$ et la non structuration du marché $(22,81 \%)$. Les relations entretenues entre les différents acteurs sont plus d'ordre social (20,49\% parental, $61,48 \%$ amical, $13,11 \%$ les deux) que professionnel $(4,92 \%)$. Les prix varient suivant les acteurs et le département. Le prix du poulet aux consommateurs est élevé étant donné qu'il passe par plusieurs intermédiaires et que chacun d'eux cherche à avoir un bénéfice. Le prix d'achat chez le producteur varie de 1480 à 2206 Fcfa avec une moyenne de 1947,68 Fcfa et celui de vente au consommateur de 4040 à $4430 \mathrm{Fcfa}$ avec une moyenne de 4216,33 Fcfa.

L'origine de l'information sur la disponibilité du produit est constituée par la demande de l'acheteur aux producteurs de porte à porte dans différents villages $(63,9 \%)$ ou par appel téléphonique $(8,2 \%)$. Vingtquatre virgule six pour cent $(24,6 \%)$ des acteurs ont affirmé que ce sont les producteurs qui leur livrent l'information à la maison ou au marché. La commercialisation des poulets du pays dans la région de Kédougou est informelle et les poulets sont vendus à l'état vivant. La distance moyenne entre la concession de l'acheteur et le lieu d'achat est de $26,65 \pm 20,07 \mathrm{~km}$. La distance la plus courte est à Salémata $(16,23 \pm 15,31 \mathrm{~km})$ et la plus 
éloignée à Saraya $(38,93 \pm 24,32 \mathrm{~km})$. Par contre, la distance moyenne entre la concession du vendeur et le lieu de vente est de 34,5 $\pm 35,36 \mathrm{~km}$ et la plus éloignée se trouve dans le département de Salémata $(40,23 \pm 35,38 \mathrm{~km})$. La fréquence moyenne d'achat des poulets par acteur et par semaine

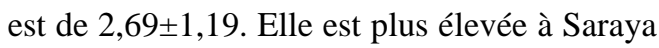
$(4,18 \pm 1,5)$ et moins importante à Kédougou $(1,64 \pm 0,62)$. La fréquence moyenne de vente des poulets par acteur et par semaine est de $2,42 \pm 1,65$. Quant aux effectifs moyens des poulets par achat et par vente, ils varient, respectivement, de $22,74 \pm 16,67$ à $26,47 \pm 34,32$ avec une moyenne de $24,81 \pm 22,1$ et de $17,89 \pm 13,86$ à $37,47 \pm 57,23$ avec une moyenne de $25,62 \pm 28,35$ par acteur (Tableau 2).

Les périodes de grande vente dans tous les départements dépendent des circonstances. Elles sont constituées principalement de jours de fête $(83,1 \%)$ et la saison hivernale $(8,45 \%)$.

\section{Mode de transport et technique d'emballage}

Quatre modes de transport (véhicule, moto, vélo, à pied) et deux techniques d'emballage sont utilisés par les différents acteurs (Figure 2). Les moyens de transport les plus utilisés sont la bicyclette $(47,75 \%)$ et la motocyclette $(23,6 \%)$. Dix-huit virgule quatre-vingt-quatre pour cent $(18,84 \%)$ des commerçants pratiquent le commerce des poulets à pied et le reste $(9,82 \%)$ à véhicule. Les techniques d'emballage employées sont le panier $(47,09 \%)$ et le cageot $(19,42 \%)$. Par contre, $33,49 \%$ des acteurs n'utilisent aucune technique d'emballage.

\section{Modes de règlement}

Trois modes de règlement sont utilisés dans le circuit de la commercialisation du poulet du pays (Tableau 3). Dans tous les trois départements, c'est le règlement au comptant qui domine dans n'importe quelle activité.
Seulement $10,66 \%$ des acteurs financent leurs activités de commerce à partir du capital obtenu par crédit. Ce financement provient soit d'un parent $(53,85 \%)$ soit d'une banque $(46,15 \%)$. Elle est à $100 \%$ bancaire et parentale à Salémata et à Kédougou, respectivement. Le remboursement est mensuel $(15,38 \%)$, trimestriel $(30,77 \%)$, semestriel $(30,77 \%)$ ou annuel $(15,38 \%)$. Par contre, 7,69\% des acteurs ont affirmé que le remboursement se fait juste après la vente et que le bénéfice est partagé directement en deux. Vingt-deux virgule quatre-vingt-quinze pour cent $(22,95 \%)$ des enquêtés vendent d'autres animaux dont les petits ruminants $(53,57 \%)$, les grands ruminants $(21,43 \%)$ ou les deux (25\%) dans le but d'avoir plus de revenus $(100 \%)$.

\section{Lieu de gardiennage des poulets}

Les commerçants gardent les poulets avant la vente à la maison $(77,62 \%)$ ou au marché $(22,38 \%)$. Ils sont gardés dans des poulaillers et/ou des cases $(53,3 \%)$, des cases et/ou caisses $(33,6 \%)$, des paniers $(6,85 \%)$ ou des endroits aménagés à cette fin $(6,25 \%)$. Cinquante-deux virgule cinq pour cent $(52,5 \%)$ des acheteurs ne parviennent pas à écouler en totalité leurs poulets en une seule fois. Trente-quatre virgule trente-sept pour cent $(34,37 \%)$ des vendeurs ont affirmé que les poulets non vendus sont laissés au marché ou retournés à la maison $(65,63 \%)$.

\section{Analyse de la rentabilité commerciale}

La marge bénéficiaire nette moyenne par département et par acteur est significativement différente $(\mathrm{p}<5 \%)$. Sa moyenne par département et par poulet est de 340,07 Fcfa avec une valeur très élevée à Saraya (408 Fcfa). La commercialisation du poulet du pays est plus rentable pour le grossiste rural (610 Fcfa) comparé aux autres acteurs avec une valeur plus élevée (700 Fcfa) dans le département de Saraya (Tableau 4). Les revenus issus de ce commerce permettent à 
41,8\% de notre échantillon de faire face aux différentes dépenses familiales (produits alimentaires/scolaires, achat des équipements/chaussures/habits, frais scolaires/soins de santé, ...). Par contre, 58,2\% des acteurs ont affirmé que les revenus issus de cette activité leur permettent, en plus d'être à la hauteur des dépenses ponctuelles, de faire quelques économies.

\section{Difficultés liées à l'approvisionnement et à la commercialisation}

Quatre-vingt-quatre virgule quarante-trois pour cent $(84,43 \%)$ et $68,85 \%$ des acteurs de notre échantillon ont affirmé rencontrer des problèmes lors de l'achat et de la vente des oiseaux, respectivement. Pendant l'achat, ces problèmes sont principalement liés à l'insuffisance du produit et ses conséquences (vol, cherté, concurrence) $(65,08 \%)$, les maladies et/ou les mortalités $(18,25 \%)$, l'enclavement de la zone $(8,73 \%)$ ainsi que le manque de capitaux $(7,94 \%)$. Par contre lors de la vente, les difficultés sont représentées par l'enclavement de la zone source du problème de transport $(38,39 \%)$ d'une part, l'inorganisation du marché $(27,27 \%)$, les mortalités et/ou maladies $(16,16 \%)$, le manque de clients $(12,79 \%)$ et le manque de capitaux $(5,39 \%)$, d'autre part.

Tableau 1 : Répartition des acteurs par département.

\begin{tabular}{lccccc}
\hline \multirow{2}{*}{ Acteurs } & \multicolumn{3}{c}{ Départements } & Effectif & Pourcentage \\
\cline { 2 - 4 } & Salémata & Kédougou & Saraya & Total & \\
\hline Producteurs & 5 & 3 & 3 & 11 & 9,02 \\
Intermédiaire I & 8 & 22 & 13 & 43 & 35,25 \\
Intermédédiaire II & 7 & 19 & 10 & 36 & 29,51 \\
grossistes ruraux & 2 & 5 & 3 & 10 & 8,2 \\
Grossistes urbains & 0 & 3 & 0 & 3 & 3,28 \\
Détaillants & 3 & 11 & 5 & 19 & 15,57 \\
Total & 25 & 62 & 35 & 122 & 100 \\
Pourcentage & 20,49 & 50,82 & 28,69 & 100 & \\
\hline
\end{tabular}

Tableau 2 : Fréquence, effectif et distance entre lieu d'achat et vente.

\begin{tabular}{llrlrc}
\hline Paramètres & & Salémata & \multicolumn{1}{c}{ Kédougou } & \multicolumn{1}{c}{ Saraya } & \multicolumn{1}{c}{ Moyenne } \\
\hline $\begin{array}{l}\text { Distance } \\
\text { moyenne : }\end{array}$ & lieu d'achat & $16,23 \pm 15,31$ & $24,79 \pm 20,58$ & $38,93 \pm 24,32$ & $26,65 \pm 20,07$ \\
concession & vente & $40,23 \pm 35,38$ & $25,64 \pm 25,52$ & $37,44 \pm 45,18$ & $34,5 \pm 35,36$ \\
Fréquence par & Achat & $2,54 \pm 1,44$ & $1,64 \pm 0,62$ & $4,18 \pm 1,5$ & $2,69 \pm 1,19$ \\
semaine & Vente & $3,00 \pm 1,92$ & $1,79 \pm 1,07$ & $2,48 \pm 1,96$ & $2,42 \pm 1,65$ \\
Effectif par & Achat & $25,21 \pm 15,32$ & $22,74 \pm 16,67$ & $26,47 \pm 34,32$ & $24,81 \pm 22,1$ \\
semaine & Vente & $21,51 \pm 13,96$ & $17,89 \pm 13,86$ & $37,47 \pm 57,23$ & $25,62 \pm 28,35$ \\
\hline
\end{tabular}




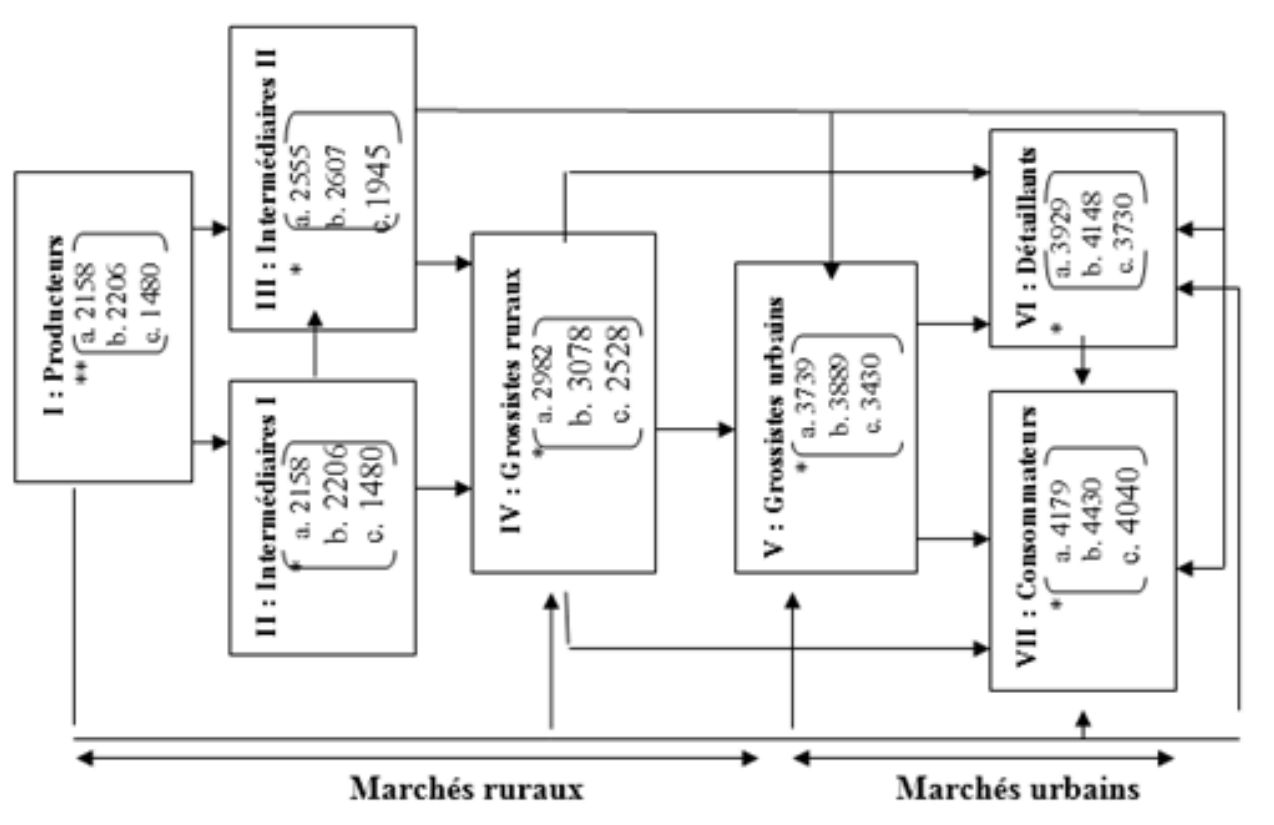

Figure 1 : Acteurs, prix, niveau et circuits de la commercialisation.

I, ..., VII : niveaux des différents acteurs dans le circuit de la commercialisation **: prix de vente par le producteur ; *: Différents prix d'achat par département et par acteur ( $a:$ Salémata ; $b:$ Kédougou ; $c:$ Saraya)

Extrémité de la flèche : acheteur (acteur) et prix de vente du poulet à l'acteur du niveau directement supérieur; Origine de la flèche : vendeur et le prix à payer par l'acteur du niveau directement supérieur.

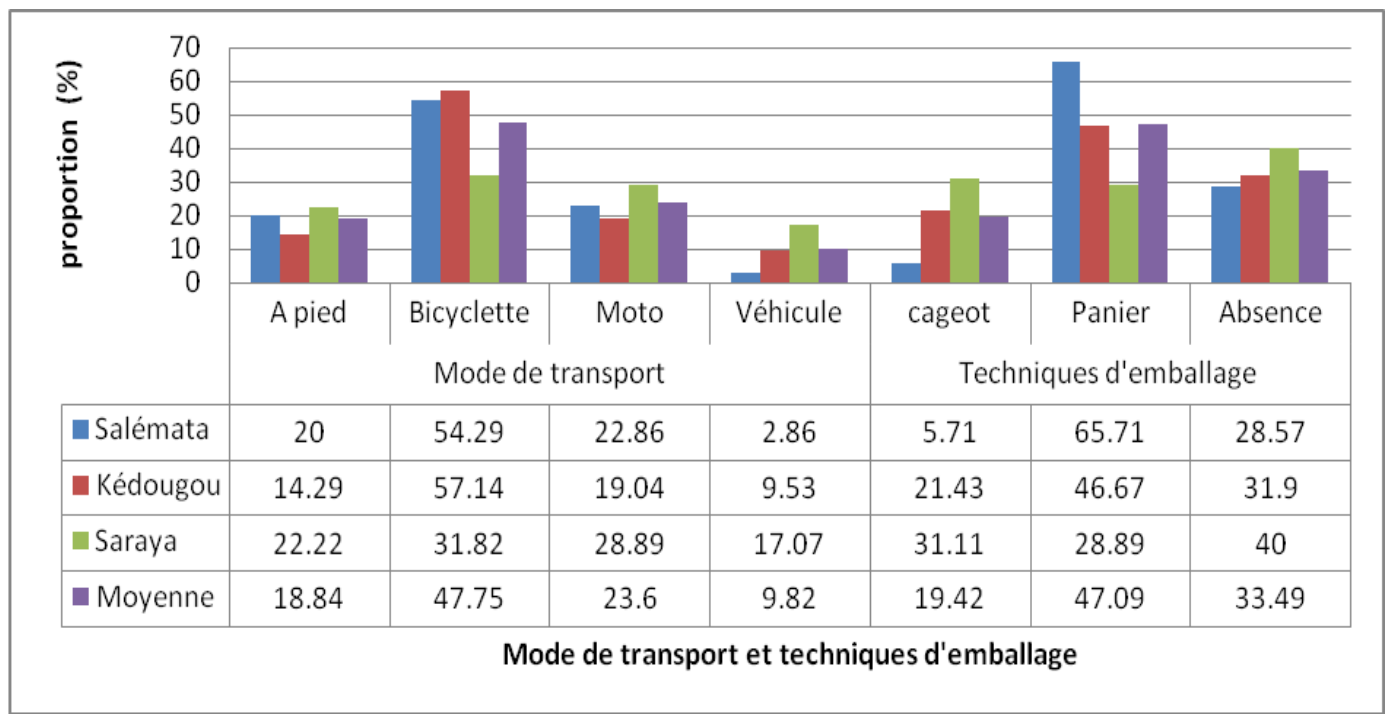

Figure 2 : Mode de transport et techniques d'emballage. 
Tableau 3 : Modes de règlement.

\begin{tabular}{lccccccccc}
\hline \multirow{2}{*}{ Département } & \multicolumn{3}{c}{ Règlement Achat } & \multicolumn{3}{c}{ Règlement Transport } & \multicolumn{3}{c}{ Règlement Vente } \\
\cline { 2 - 9 } & I & II & III & I & II & III & I & II & III \\
\hline Salémata & 88,57 & 2,86 & 8,57 & 22,86 & 8,57 & 8,57 & 74,26 & 8,57 & 14,29 \\
Kédougou & 61,9 & 4,76 & 33,33 & 23,81 & 0 & 0 & 69,05 & 0 & 30,95 \\
Saraya & 84,09 & 0 & 15,91 & 42,22 & 0 & 0 & 95,56 & 0 & 2,22 \\
Moyenne & $\mathbf{7 8 , 1 7}$ & $\mathbf{2 , 5 4}$ & $\mathbf{1 9 , 2 7}$ & $\mathbf{2 9 , 6 3}$ & $\mathbf{2 , 8 6}$ & $\mathbf{2 , 8 6}$ & $\mathbf{8 1 , 0 8}$ & $\mathbf{2 , 9 4}$ & $\mathbf{1 5 , 9 8}$
\end{tabular}

I: au comptant; II: à crédit; III: les deux (au comptant et à crédit).

Tableau 4 : Marge Bénéficiaire nette par acteur et par poulet entre deux acteurs successifs.

\begin{tabular}{|c|c|c|c|c|c|c|}
\hline \multirow[b]{2}{*}{ 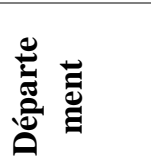 } & \multicolumn{6}{|c|}{ Marge Bénéficiaire nette par acteur et par poulet entre deux acteurs successifs } \\
\hline & $\begin{array}{c}\text { Intermédiaire } \\
\text { I }\end{array}$ & $\begin{array}{c}\text { Intermédiaire } \\
\text { II }\end{array}$ & $\begin{array}{l}\text { Grossiste } \\
\text { rural }\end{array}$ & $\begin{array}{c}\text { Grossiste } \\
\text { urbain }\end{array}$ & Détaillants & moyenne \\
\hline Salémata & $160 \pm 17,11$ & $190 \pm 17,83$ & $520 \pm 23,08$ & $340 \pm 13,01$ & $150 \pm 21,51$ & $272 \pm 7,62^{\mathrm{a}}$ \\
\hline Kédougou & $200 \pm 22,97$ & $270 \pm 15,12$ & $610 \pm 11,24$ & $440 \pm 14,55$ & $181 \pm 19,35$ & $340,2 \pm 8,17^{b}$ \\
\hline Saraya & $163 \pm 16,08$ & $381 \pm 24,81$ & $700 \pm 18,42$ & $510 \pm 14,08$ & $286 \pm 11,49$ & $408 \pm 7,23^{c}$ \\
\hline Moyenne & $174,33 \pm 18,72^{d}$ & $280,33 \pm 19,26^{\mathrm{e}}$ & $610 \pm 17,28^{f}$ & $430 \pm 13,88^{g}$ & $205,67 \pm 17,47^{\mathrm{h}}$ & $\mathbf{3 4 0 , 0 7}$ \\
\hline
\end{tabular}

Les moyennes suivies de lettres différentes (ligne ou colonne) sont différentes statistiquement à $5 \%$.

\section{DISCUSSION}

Il ressort de cette étude que la commercialisation des poulets du pays est pratiquée par des hommes. Des résultats similaires ont été trouvés par Dièye et al. (2010) au Sénégal, Gondwe et al. (2005) au Malawi et par Issa et al. $(2012,2015)$ au Tchad. Néanmoins, nos résultats sont en désaccord avec les constats rapportés par Aklilu et al. (2007) et Dinka et al. (2010) en Ethiopie, Emuron et al. (2010) en Ouganda, Guèye (2003) au Botswana, Alabi et al. (2006) au Nigéria et Sodjinou (2011) au Bénin. En effet, ces différents auteurs avaient signalé que l'activité de vente de volailles sur les marchés était dominée par les femmes. La forte présence d'hommes dans le circuit pourrait découler des prix de vente élevés observés et l'accaparement des activités féminines par les hommes quand leur rentabilité augmente (Traoré, 2005; Seye, 2007).

Les raisons de changement d'activités observées dans la présente étude confirment les constats rapportés par Aklilu et al. (2007). En effet, d'après ces auteurs, la commercialisation des volailles est l'une des rares occasions pour les ruraux de générer rapidement des revenus en espèces pour subvenir à leurs divers besoins.

La diversité des modes de transport dans la présente étude corrobore les constats rapportés par Byarugaba (2007) et par Emuron et al. (2010) en Ouganda. Par ailleurs, 
Gondwe et al. (2005) et Emuron et al. (2010) avaient signalé que les personnes qui se rendaient au marché hebdomadaire utilisent des moyens de transport différents. C'est pourquoi nous nous mettons d'accord avec Byarugaba (2007) pour affirmer qu'il n'existe pas de moyens spécialisés pour le transport des poulets locaux en Afrique subsaharienne.

Le transport à bicyclette est le mode le plus utilisé dans notre étude. Ce même constat a été fait au Malawi (Gondwe et al., 2005). La prépondérance du transport à bicyclette, à pied et à motocyclette observé dans cette étude serait due à la courte distance se trouvant entre la maison et le lieu d'achat et/ou de vente. Cela confirme les observations énoncées par Mopate et Djimet (2012) et Emuron et al. (2010) selon lesquelles les acteurs dans la commercialisation utilisent les moyens de transport appropriés lorsque les distances sont longues.

La diversité des acteurs dans le circuit de la commercialisation dans la présente étude confirme les enregistrements faits en Tanzanie (Mlozi et al., 2003), au Malawi (Gausi et al., 2004), en Afrique de l'ouest (Williams et al., 2006; Bâ, 2010 ; Teno, 2010 ; Sikangueng Mbouga, 2011) et en Ethiopie (Dessie et Ogle, 2001). En effet, ces auteurs avaient remarqué qu'il existe une chaîne d'intermédiaires dans la commercialisation de poulets locaux entre le producteur et le consommateur final. Ce même constat a été rapporté par Malam Boukar et al. (2016) pour les principaux produits agricoles des cuvettes oasiennes du département de Gouré au Niger.

Le système de commercialisation que nous avons observé est le même que celui rapporté par Gausi et al. (2004) au Malawi, par Sodjinou (2011) au Bénin et par Moges et Dessie (2010) en Ethiopie. Toutefois, il diffère de celui rapporté par Sikangueng Mbouga (2011) sur l'axe Louga-Dakar et par Bâ (2010) au Sénégal même si les acteurs dans le circuit de la commercialisation sont les mêmes. En effet, chez ce dernier auteur, les producteurs ne vendent pas leurs poulets à tous les acteurs du marché urbain, ce qui n'est pas le cas dans la présente étude. Par contre, pour Sikangueng Mbouga (2011), à part que les producteurs ne vendent jamais leurs produits à aucun des acteurs du marché urbain, même les intermédiaires II ne livrent pas leurs poulets à tous les acteurs de ce même marché, contrairement à nos observations.

Nous avons observé un système de commercialisation informel similaire à celui décrit en Ouganda (Emuron et al., 2010; Natukunda et al., 2011), au Burkina Faso (Ouedraogo et Zoundi, 1999) et au Sénégal (Guèye et al., 1998). Les critères de fixation du prix dans la présente étude sont les mêmes que ceux rapportés par Halima (2007) en Ethiopie.

Le prix du poulet varie d'un marché à l'autre et d'un acteur à l'autre. Le niveau élevé du prix au consommateur rapporté dans la présente étude est dû, en accord avec Williams et al. (2006) et Emuron et al. (2010), au fait que l'implication de beaucoup d'intermédiaires dans le commerce de poulet local augmente les coûts et les risques dans le processus de commercialisation. En effet, chaque intermédiaire essaie de se faire un bénéfice à son niveau. De même, un long circuit ainsi que le mode de transport et de stockage des oiseaux peuvent être à la base des mortalités et/ou maladies. Par ailleurs, d'après MacCaluin et al. (2003), le fait d'utiliser des modes de transport et techniques d'emballage des poulets inappropriés en parcourant une longue distance les rend plus vulnérables ce qui peut entraîner beaucoup de pertes. Le même problème est valable pour les acteurs qui se déplacent à pied. A cela s'ajoute le fait que durant le transport, les oiseaux ne sont ni alimentés ni abreuvés. En outre, nous nous accordons avec Emuron et al. (2010) ainsi qu'avec Tabba et Alshawabkeh (2000) que le confinement des oiseaux au cours du transport ainsi que leur étalement sur le lieu d'achat/vente alors que ces oiseaux 
proviennent de différents producteurs et qu'il n'y a pas de systèmes de collecte des fientes les exposent aux différentes sources d'infection avec des risques non négligeables de mortalité.

La proportion élevée des acteurs qui n'utilisent pas l'emballage $(33,49 \%)$ est une conséquence des techniques de transport (bicyclette, motocyclette, piéton) utilisées par la majorité de nos acteurs dans le circuit de commercialisation.

Le prix observé au consommateur dans la présente étude est supérieur à celui rapporté par Teno (2010) et Sikangueng Mbouga (2011) à Dakar (Sénégal). Cette différence des prix pourrait être due aux différentes charges liées à l'activité, à la présence de beaucoup de sites d'orpaillage et enfin à l'enclavement de la zone qui réduit l'accès au marché.

Les contraintes dans la commercialisation des poulets observées dans la présente étude sont presque les mêmes que celles rapportées au Kenya (Kaudia et Kitayi, 2002), en Ouganda (Emuron et al., 2010 ; Nakatunda et al., 2011), au Malawi (Gausi et al., 2004) et au Tchad (Issa et al., 2015).

\section{Conclusion}

Il ressort de cette étude que la commercialisation des poulets du pays implique plusieurs acteurs qui empruntent des circuits courts ou longs en utilisant divers moyens de transport. Bien que les circuits commerciaux traditionnels soient adaptés aux caractéristiques de la production, ils présentent un certain nombre d'aspects négatifs au niveau du transport, du stockage et des circuits même de commercialisation. Ainsi, la vente directe, la réorganisation des circuits commerciaux et des acteurs ainsi que l'amélioration des conditions de transport amélioreraient le système d'approvisionnement et de commercialisation du poulet villageois au Sénégal. Le système de commercialisation, bien qu'informel, constitue un excellent moyen pour renforcer la sécurité alimentaire et améliorer les revenus des aviculteurs villageois.

\section{CONFLITS D'INTERETS}

Les auteurs déclarent qu'il n'existe aucun conflit d'intérêts concernant la publication de cet article.

\section{CONTRIBUTIONS DES AUTEURS}

GN a contribué à la rédaction du protocole d'étude ; AM, SBA et WO ont contribué à son amélioration. Tous les auteurs ont participé à la rédaction du présent article. Tous les auteurs ont participé à la rédaction du présent article.

\section{REMERCIEMENTS}

Les auteurs remercient le Projet de Développement de l'Elevage au Sénégal Oriental et en Haute-Casamance (PDESOC) pour avoir mis à leur disposition les moyens nécessaires pour faciliter et mener à terme les enquêtes.

\section{REFERENCES}

Aklilu HA, Almekinders CJM, Udo HMJ, Van Der Zijpp AJ. 2007. Village poultry consumption and marketing in relation to gender, religious festivals and market access. Tropical Animal Health and Production, 39(3):165-177. DOI: 10.1007/s11250-007-9002-8

Alabi RA, Esobhawan AO, Aruna MB. 2006. Econometric determination of the contribution of the family poultry to the women's income in the Niger- Delta, Nigeria. Journal of Central European Agriculture, $\quad$ 7(4):753-760. http://www.agr.hr/jcea/issues/jcea74/pdf/jcea74-21.pdf

Bâ M. 2010. Économie de la filière avicole semi-industrielle au Sénégal : typologie des cicuits de commercialisation. Rapport COTAVI, Dakar (Sénégal), 51 p

Byarugaba DK. 2007. The structure and importance of the commercial and village based poultry industry in Uganda, 
A consultancy report for FAO, Kampala, $54 \mathrm{p}$

Dieye PN, Missohou A, Faye A. 2010. L'aviculture familiale: un levier pour améliorer les revenus des éleveurs pauvres au Sud du Sénégal. In L'Elevage: Richesse des Pauvres, Faye B, Duteurtre G (eds). Editions Quae: Paris ; 191-201.

Dinka H, Chala R, Dawo F, Leta S, Bekana E. 2010. Socio-economic importance and management of village chicken production in rift valley of Oromia, Ethiopia. Livestock Research for Rural Development, 22(203). Retrieved October 26, 2015, from http://www.lrrd.org/lrrd22/11/dink22203 .htm

Emuron N, Magala H, Kyazze FB, Kugonza DR, Kyarisiima CC. 2010. Factors influencing the trade of local chickens in Kampala city markets. Livestock Research for Rural Development, 22(76). Retrieved October 23, 2015, from

http://www.lrrd.org/lrrd22/4/emur22076. $\mathrm{htm}$

Gausi JCK, Safalaoh ACL, Banda JW, Ng'ong'ola DH. 2004. Characterisation of the smallholder poultry marketing systems in rural Malawi: A case study of Malingunde Extension Planning Area. Livestock Research for Rural Development, 16(97). Retrieved October 24, 2015, from http://www.lrrd.org/lrrd16/12/gaus16097 .htm

Gondwe TN, Wollny CBA, Kaumbata W. 2005. Marketing system and channels for scavenging local chickens in Lilongwe, Malawi. Livestock Research for Rural Development, 17((24). Retrieved October 26, 2015, from http://www.lrrd.org/lrrd17/3/gond17024. htm

Gueye EF. 2003. Poverty alleviation, food security and the well-being of the human production through family poultry in low-income food-deficit countries. Food, Agriculture and Environment, 1(2): 1221

Guèye EF, Ndiaye A, Branckaert RDS. 1998. Prediction of body weight on the basis of body measurements in mature indigenous chickens in Senegal. Livestock Research for Rural Development, 10(28). Retrieved October 25, 2015, from http://www.lrrd.org/lrrd10/3/sene103.ht $\mathrm{m}$

Halima HM. 2007. Phenotypic and genetic characterization of indigeneous chicken population in Northwest Ethiopia. PhD Thesis, University of the Free State, Bloemfontein, South Africa.

Hellin J, Griffith A, Mike A. 2005. Mapping the market: market-literacy for agricultural research and policy to tackle rural poverty in Africa. Beyond Agriculture-Making Markets Work for the Poor, Proceedings of an International Seminar 28 February-1 March 2005, Westminster, London, UK, 110-150

Issa YA, Mopate LY, Zeuh V, Ardjoun KD, Bada AO, Adam BM. 2015. Supply and Marketing of Local Chicken in the Town of Abeche in Eastern Chad. Pakistan Journal of Nutrition, 14(1): 6-12. DOI: 10.3923 / pjn.2015.6.12

Issa $\mathrm{Y}$, Mopate Logtene $\mathrm{Y}$, Djougui $\mathrm{S}$, Missohou A. 2012. Tradidional poultry supply and marketing in the city of N'Djamena in Chad. International Journal of Poultry Science, 11(5): 341348. DOI: $10.3923 /$ ijps.2012.341.348

Kaudia TJ, Kitalyi AJ. 2002. The Bangladesh model and other experiences in family poultry development: Commercializing rearing of village chicken in Kenya. INFPDE. Conferences International Network for Family Poultry Development (INFPD). www.fao.org/ag/AGAinfo/themes/en/inf pd/econf_bang.html , pp 116-120 
Kugonza DR, Kyarisiima CC, Iisa A. 2008. Indigenous chicken flocks of Eastern Uganda: I. Productivity, management and strategies for better performance. Livestock Research for Rural Development, 20(137). Retrieved November 6, 2015, from http://www.lrrd.org/lrrd20/9/kugo20137. $\mathrm{htm}$

MacCaluim JM, Abeyesingh SM, White RP, Wathes CM. 2003. A continuouschoice assessment of the domestic fowl's aversion to concurrent transport stressors. Animal Welfare, 12: 95-107.

Malam Boukar AK, Tidjani AD, Yamba B, Lebailly P. 2016. Performance et circuit de commercialisation des principaux produits agricoles des cuvettes oasiennes du département de Gouré (Niger). Int. J. Biol. Chem. Sci., 10(5): 2202-2214. http://dx.doi.org/10.4314/ijbcs.v10i5.21

Mlozi MRS, Kakengi AVM, Minga UM, Mtambo AM, Olsen JE. 2003. Marketing of free range local chickens in Morogoro and Kilosa urban markets, Tanzania. Livestock Research for Rural Development, 15(14). Retrieved October 24, 2015, from http://www.lrrd.org/lrrd15/2/mloz152.ht $\mathrm{m}$

Moges F, Dessie T. 2010. Characterization of village chicken and egg marketing systems of Bure district, North-West Ethiopia. Livestock Research for Rural Development, 22196). Retrieved October 25, 2015, from http://www.lrrd.org/lrrd22/10/moge2219 6.htm

Mopate LY, Djimet M. 2012. Approvisionnement du marché et transformation hors-foyer de la volaille traditionnelle dans la ville de Bongor (Tchad). Revue Scientifique du Tchad (RST), 11(1 spécial): 63-73

Mwalusanya NA, Katule AM, Mutayoba SK, Mtambo MMA. 2002. Productivity of local chickens under village management conditions. Tropical Animal Health and Production, 34: 405-416. DOI: 10.1023 / A: 1020048327158

Natukunda K, Kugonza DR, Kyarisiima CC. 2011. Indigenous chickens of the Kamuli Plains in Uganda: II. Factors affecting their marketing and profitability. Livestock Research for Rural Development, 23(221). Retrieved October 25, 2015, from http://www.lrrd.org/lrrd23/10/natu23221. htm

Niang M, Mbaye M. 2013. Evolution des exportations de bétail malien au Sénégal suite aux récentes crises. Rapport final, Dakar/Sénégal, $37 \mathrm{p}$

Okot MW. 1990. A co-operative approach to smallholder rural poultry production in Uganda. In: Smallholder Rural Poultry Production. Proceedings of a CTA Seminar, Thessalonica, Greece, October, 9-13, 249-253

Ossebi W. 2011. Analyse de la filiere « poulet du pays »au Sénégal : aspects économiques et organisationnels. Mémoire de Master, EISMV, Dakar, 13

Seye EM. 2007. Evaluation d'un transfert de Paquet technique en Aviculture familiale et de son impact sur la génération de revenus et l'égalité du genre. Thèse : Méd Vét, Dakar; 12

Sikangueng Mbouga FC. 2011. Etude de la commercialisation du poulet local au Sénégal : acteurs, marches, circuits et systèmes de transport sur l'axe Louga Dakar. Mémoire de Master, EISMV, Dakar, 16

Sodjinou E. 2011. Poultry-based intervention as tool for poverty reduction and gender empowerment: Empirical evidence from Benin. PhD Thesis, Institute of Food and Resource Economics, University of Copenhagen, Denmark, 239 p

Tabba MJ, Alshawabketh K. 2000. Some factors affecting pre-slaughtering mortality and damage to broilers and interaction during transportation to 
processing plants. Dirasat Agricultural Science, 27: 375-384

Teno G. 2010. Analyse du système de commercialisation du poulet du pays dans le département de Dakar (Sénégal). Mémoire de Master, EISMV, Dakar, 03

Traore M. 2005. Evaluation de l'impact d'un transfert de paquet technique (amélioration génétique et des conditions d'élevage) sur la génération de revenus en aviculture traditionnelle dans les Niayes. Thèse Méd. Vét., Dakar, 23

Traoré EH. 2001. Amélioration de la production avicole en élevage familial traditionnel à travers une meilleure gestion des contraintes de production et de Santé animale TCP/SEN 065. Phase I: 2001-2002 in "Système de production aviaire » Projet FAO. Rapport de mission, $36 \mathrm{p}$

Williams TO, Spycher B, Okike I. 2006. Improving livestock marketing and intraregional trade in West Africa: Determining appropriate economic incentives and policy framework. ILRI (International Livestock Research Institute), Nairobi, Kenya, 122 p. 